\title{
Magnetic field as a probe of gap energy scales in YBCO
}

\author{
N. D. Whelan and J. P. Carbotte \\ Department of Physics and Astronomy, McMaster University, Hamilton, Ontario, Canada L8S $4 M 1$
}

(September 10, 2018)

\begin{abstract}
Among high $\mathrm{T}_{c}$ materials, the $\mathrm{YBCO}\left(\mathrm{YBa}_{2} \mathrm{Cu}_{3} \mathrm{O}_{7-x}\right)$ compounds are special since they have superconducting chains as well as planes. We show that a discontinuity in the density of states as a function of magnetic field may appear at a new energy scale, characteristic of the chain and distinct from that set by the $d$ wave gap. This is observable in experimental studies of the thermodynamical properties of these systems, such as the specific heat.
\end{abstract}

It is widely accepted that a $d$-wave pairing gap describes the superconductivity in high $\mathrm{T}_{c}$ compounds [1]. The YBCO compounds are special however, due to the fact that the one dimensional chains change the Fermi surface, giving a quasi-linear branch, and thereby introducing a new energy scale for the variation of the gap on the Fermi surface. We argue that this can be directly measured using a magnetic field.

Scanning tunnelling microscopy (STM) measurements [2,3] of the local density of states of YBCO agree with earlier tunnelling measurements [4] and show a very clear structure around $20 \mathrm{meV}$ corresponding to the full gap energy. They also indicate structure at around $5.5 \mathrm{meV}$ which is absent in BSCCO compounds [3]. It is natural to assume that this is because YBCO has a different electronic structure, possessing orthorhombic chains as well as tetragonal planes. The chains introduce a new energy scale, the value of the gap function at the endpoint of the chain Fermi surface on the boundary of the Brillouin zone. Since experiments are only sensitive to the value of the gap function on the Fermi surface (and not to its global behaviour throughout the Brillouin zone) this energy scale will be apparent in experiments. Another interpretation of the additional structure is localised quasiparticle states in the vortex core [5]. Even if present, these should have a negligible effect on the bulk density of states, a point also made in Ref. [6].

We model this by assigning the chains an elliptical Fermi surface but with a highly modified gap function

$$
\Delta_{\mathbf{k}}=\Delta_{0}(\cos 2 \phi+s)
$$

$\phi$ is the polar coordinate in $\mathbf{k}$ space and the dimensionless parameter $s$ (with $|s|<1$ ) indicates the effective amount of $s$ wave in the order parameter. We stress that (11) is not the gap function defined throughout the Brillouin zone but is rather its value evaluated on the Fermi surface. Along the chain Fermi surface the gap function will have a dependence like (11) even if it is globally pure $d$-wave. This is an effective theory to model the contribution of the chains to the density of states and reflects a combination of gap symmetry and Fermi surface geometry. (Independent information on the existence of a subdominant $s$-wave component is provided by Joseph- son tunnelling data $[7,8]$.)

Standard theory for the density of states gives [9]

$$
\frac{N(\omega)}{\bar{N}} \propto \int \mathrm{d} \mathbf{k} \operatorname{Im}\left\{\frac{|\omega|}{\omega^{2}-\Delta_{\mathbf{k}}^{2}-\xi_{\mathbf{k}}^{2}}\right\},
$$

where $\xi_{\mathbf{k}}$ is the dispersion relation for the quasi-particle excitation energy (for the moment we take it $\xi_{k}=\mathbf{k}^{2} / 2 m$ with $\mathbf{k}$ the momentum and $m$ the effective mass) and $\bar{N}$ is the normal, nonsuperconducting density of states. Using the gap function (11), this integral is easily done and is plotted in Fig. 目 using $s=0.57$ (this choice is explained below.) There is a van Hove singularity at $\omega=(1+|s|) \Delta_{0}$ as we expect 10], however there is another at $(1-|s|) \Delta_{0}$, in qualitative agreement with [2, 3].

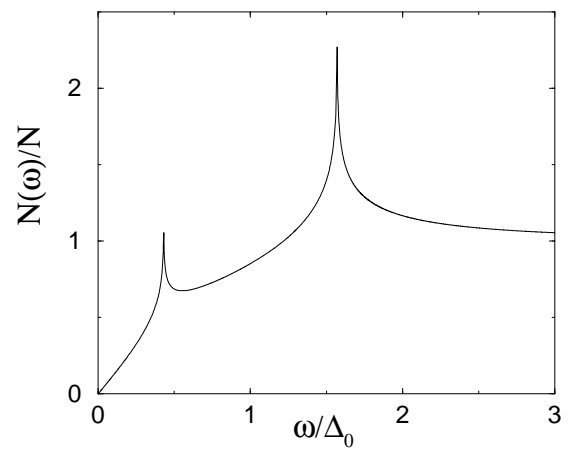

FIG. 1. Typical result for $N(\omega)$ for a pure superconductor using $s=0.57$ to introduce a second low energy scale. Note the van Hove singularities at $\omega / \Delta_{0}=(1 \pm|s|)$.

A problem with the measured $N(\omega)$ is that there is a large zero bias anomaly in contradiction with theory and presumably arising from surface effects. It is therefore useful and possibly better to measure a bulk property. We propose the low temperature limit of the specific heat, which comes from the density of quasiparticle states at zero frequency. We focus on the magnetic field regime $H_{c 1} \ll H \ll H_{c 2}$ where the density of states is given by quasi-particle excitations in the presence of the induced vortices. For each magnetic field, there is a magnetic energy, which scales as $\sqrt{H}$ [11,12]. We expect structure at critical fields for which the magnetic energy sweeps through the singularities at $\Delta_{0}(1 \pm s)$ in Fig. 1. The new, lower energy scale is well within the range of available 
fields and is observable in specific heat experiments which probe the vortex modified density of states.

In the presence of the vortex, the Cooper pairs have a superfluid velocity $\mathbf{v}_{\mathbf{s}}$ which depends inversely on the distance to the vortex core. The quasi-particle energies of momentum $\mathbf{k}$ are Doppler shifted by $\omega \rightarrow \omega+\mathbf{v}_{\mathbf{s}} \cdot \mathbf{k}$ [13], which can be thought of as introducing a spatially dependent shift in chemical potential. We are interested in the zero frequency case, so we can use Eq. (2) but with $\omega$ replaced by $V=\mathbf{v}_{\mathbf{s}} \cdot \mathbf{k}$. In addition to the trace integral over $\mathbf{k}$, we also do a spatial average within the vortex unit cell, with coordinates $\mathbf{r}$ :

$$
N_{0}(H) \propto \int \mathrm{d} \mathbf{k} \int \mathrm{d} \mathbf{r} V \delta\left(\zeta_{\mathbf{k}}^{2}-V^{2}+\Delta_{\mathbf{k}}^{2}\right) .
$$

To find $\mathbf{v}_{\mathbf{s}}$, we use the free energy density per unit length

$$
F=\int \mathrm{d}^{2} r\left(B^{2}+\lambda_{x}^{2}(\nabla \times B)_{x}^{2}+\lambda_{y}^{2}(\nabla \times B)_{y}^{2}\right) .
$$

We have introduced the parameters $\lambda_{i}^{2}=\mu_{i} \lambda^{2}$ with $\lambda^{2}=M c^{2} / 4 \pi e^{2} n_{s}, \mu_{i}=m_{i} / M$ and $M=\sqrt{m_{x} m_{y}}(\lambda$ is a mean London penetration depth and $n_{s}$ the superfluid density.) Following standard free energy minimisation 13] with a flux line source at the origin, we express the magnetic field as a modified Bessel function. In the magnetic field regime considered here, the typical spacing between vortices is much smaller than $\lambda$ and we use the small argument approximation:

$$
B \approx-\frac{\Phi_{0}}{2 \pi \lambda^{2}} \log \left(\frac{1}{\lambda} \sqrt{\frac{x^{2}}{\mu_{y}}+\frac{y^{2}}{\mu_{x}}}\right),
$$

pointing in the $\mathrm{z}$ direction and $\Phi_{0}=\pi \hbar c / e$ is the flux quantum. Applying Ampère's law, the current is

$$
\begin{aligned}
& j_{x} \approx-\frac{c \Phi_{0}}{8 \pi^{2} \lambda^{2}} \frac{1}{\frac{x^{2}}{\mu_{y}}+\frac{y^{2}}{\mu_{x}}} \frac{y}{\mu_{x}} \\
& j_{y} \approx \frac{c \Phi_{0}}{8 \pi^{2} \lambda^{2}} \frac{1}{\frac{x^{2}}{\mu_{y}}+\frac{y^{2}}{\mu_{x}}} \frac{x}{\mu_{y}} .
\end{aligned}
$$

We find $\mathbf{v}_{\mathbf{s}}$ by dividing the current by $e n_{s}$.

The quasi-particle excitations are given by the dispersion relation

$$
\zeta_{\mathbf{k}}=\frac{\hbar^{2} k_{x}^{2}}{2 m_{x}}+\frac{\hbar^{2} k_{y}^{2}}{2 m_{y}}
$$

As discussed above, we take the gap function (11) to be

$$
\Delta_{\mathbf{k}}=\Delta_{0} f(\mathbf{k})=\Delta_{0}\left(\frac{k_{x}^{2}-k_{y}^{2}}{k_{x}^{2}+k_{y}^{2}}+s\right),
$$

The analysis is simplified by a change of variables to

$$
\begin{aligned}
x^{\prime} & =x / \sqrt{\mu_{y}} & k_{x}^{\prime} & =\sqrt{\mu_{y}} k_{x} \\
y^{\prime} & =y / \sqrt{\mu_{x}} & k_{y}^{\prime} & =\sqrt{\mu_{x}} k_{y} .
\end{aligned}
$$

In terms of these new coordinates we have

$$
\begin{aligned}
\mathbf{v}_{\mathbf{s}} & =\frac{\hbar}{2 M} \frac{1}{r^{\prime}} \hat{\beta}^{\prime} \\
\zeta_{\mathbf{k}} & =\frac{\mathbf{k}^{\prime 2}}{2 M} \\
f(\mathbf{k}) & =f\left(\phi^{\prime}\right)=\frac{m_{y} / m_{x}-\tan ^{2} \phi^{\prime}}{m_{y} / m_{x}+\tan ^{2} \phi^{\prime}}+s
\end{aligned}
$$

where $\left(r^{\prime}, \beta^{\prime}\right)$ are the spatial polar coordinates $\left(\beta^{\prime}\right.$ is the vortex winding angle) and $\left(k^{\prime}, \phi^{\prime}\right)$ are the momentum polar coordinates in the new coordinates. Note that $\mathbf{v}_{\mathbf{s}} \cdot \mathbf{k}=\left|\mathbf{v}_{\mathbf{s}}\right||\mathbf{k}| \sin \left(\beta^{\prime}-\phi^{\prime}\right)$. Henceforth we drop the primes. Since we are integrating over $\beta$, we are also free to shift its origin and thereby replace $\beta-\phi$ by $\beta$. To conform to usual notation we say that the chains run along the $b$ direction which we define as parallel to the $y$ axis. Since the carrier mass in the $b$ direction is less than in the $a$ direction (due to the conductivity supplied by the chains) we conclude that $m_{y} / m_{x}<1$. In principle $s$ can have either sign (we ignore the time-reversal symmetry breaking possibility for which $s$ is complex.) For purposes of exposition we will use the realistic value $m_{y} / m_{x}=0.5$ and take $s=0.57$ so as to get a second gap scale around $5.5 \mathrm{meV}$ as seen in experiment. The oscillatory part of the gap function in (10) has been modified due to the coordinate rescaling, compared to the original $\Delta_{0} \cos 2 \phi$. Nevertheless, the amplitude has not been modified and without the $s$ term both functions would vary between plus and minus $\Delta_{0}$. Either form would yield an initial linear dependence on $\sqrt{H}[11,12$ followed by a saturation at a scale set by $\Delta_{0}$. Rather, it is the $s$ term which changes this qualitative picture. It makes the gap function have a maximum value of $\Delta_{0}(1+|s|)$ and a minimum value of $\Delta_{0}(1-|s|)$. The first of these gives the saturation scale mentioned above while the second gives structure corresponding to the first peak in Fig. 1.

We begin by nondimensionalising the spatial integral in (3). In order for the average magnetic field in the sample to equal the applied field, we require the inter-vortex spacing to be [12] $R=\sqrt{\Phi_{0} / \pi H} / a$ where $a$ is a geometrical constant of order unity which accounts for the mismatch between the circular vortices and the hexagonal lattice they fill out. We define the magnetic energy as

$$
E_{H}=\frac{a}{2 M} v_{F} \sqrt{\frac{\pi H}{\Phi_{0}}}
$$

where $v_{F}$ is the Fermi velocity and the dimensionless magnetic energy as $\nu=E_{H} / \Delta_{0}$. We then express (3) as 


$$
\frac{N_{0}(H)}{\bar{N}}=\frac{1}{2 \pi^{2}} \int_{0}^{2 \pi} \mathrm{d} \phi \int_{0}^{2 \pi} \mathrm{d} \beta \int_{0}^{1} \mathrm{~d} \rho \frac{\rho}{\sqrt{1-\left(\frac{\rho f(\phi)}{\nu \sin \beta}\right)^{2}}}
$$

where $\bar{N}$ is the normal density of states and $\rho=r / R$. We used the delta function to perform the $k$ integral and it is understood that the integration domain is limited to the range where the integrand is real.

We can do the $\rho$ and $\beta$ integrals in (12) leading to the final expression for the density of states,

$$
\frac{N_{0}(H)}{\bar{N}}=\frac{1}{2 \pi} \int \mathrm{d} \phi \min \left\{1,\left(\frac{\nu}{f(\phi)}\right)^{2}\right\} .
$$

The parameter $\nu$ is experimentally tunable while $s$ (which enters $f(\phi)$ through (10)) is a fixed, intrinsic material parameter (although it may depend somewhat on doping levels) and represents a combination of gap and Fermi surface symmetries. For the case $|s|<1$ and $\nu \ll 1$, it is simpler to go back to (12) and expand the integrand around the gap nodes. One can perform the $\phi$ integral approximately in the limit of small $\nu$ and the resulting $\beta$ and $\rho$ integrals are trivial so that

$$
\frac{N_{0}(H)}{\bar{N}} \approx\left(\frac{2}{\pi} \sum_{n} \frac{1}{\left|f_{n}^{\prime}\right|}\right) \nu .
$$

The index $n$ refers to the nodes of the gap function and $f_{n}^{\prime}$ is the derivative of the function $f$ evaluated at the nodes. This is still linear, as one has for a pure $d$-wave gap, although the presence of the $s$ term does change the positions of the gap nodes and hence affects the slope. For $|s|>1$, there are no nodes and the dependence is quadratic for small $\nu$. Since this is unphysical we do not present the explicit form.
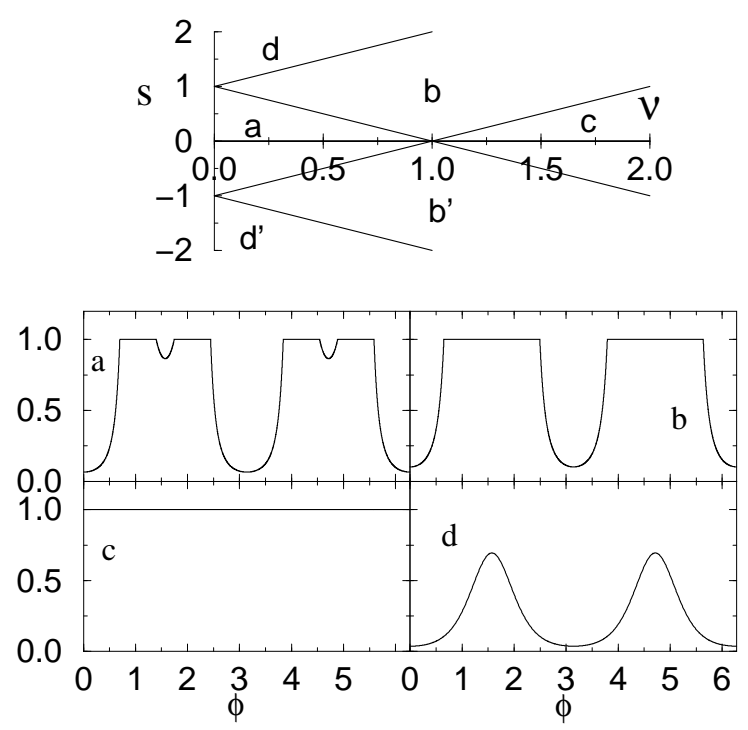

FIG. 2. The four possible behaviours of the integrand of Eq. (13) as a function of $\phi$. The values of $(\nu, s)$ are $(0.4,0.57)$, $(0.5,0.57),(2.0,0.57)$ and $(0.5,1.6)$ for cases a to $\mathrm{d}$ respectively. In all cases the mass anisotropy was $m_{y} / m_{x}=0.5$. Top: regions in the $\nu-s$ plane corresponding to the 4 types of integrands (in regions b' and d' the integrand is translated by $\pi / 2$ from its form in regions $b$ and d.)

There are four distinct behaviours of the integrand of (13), as shown in Fig. 2 and the $\nu-s$ plane is accordingly divided into six regions. For fixed $s$, we vary $\nu$ and thereby cross from one region to another. Associated with such crossings there is a corresponding nonanalyticity in the density of states, which is experimentally measurable. This is shown in Fig. 3 where we plot the density of states as a function of $\nu$ using the integral form (13). Clearly there is structure at $\nu=0.43$ and 1.57 , in accord with our general considerations. The kink at $\nu=1.57$ corresponds to the integrand saturating at unity — as we go from an integrand as in Fig. 2 $2 \mathrm{~b}$ to the type as in Fig. 2c. However, this probably lies beyond any realisable magnetic field strength. The kink at $\nu=0.43$ which corresponds to going from an integrand as in Fig. 2a to the type in Fig. 2 $2 \mathrm{~b}$ appears by eye to be a discontinuity in slope. This is not really the case, as we now discuss.

The integrand in Fig. 2a saturates around $\phi=\pi / 2$ and $\phi=3 \pi / 2$ when $\nu$ approaches $1-|s|$ and the integral does not increase as quickly after this value as it does before. We can evaluate the missing contribution, $\delta N$, from the regions around these local minima. We take $\nu$ to be just below this transition, i.e.

$$
\nu=1-|s|-\epsilon
$$

with $\epsilon \ll 1$. To leading order in $\epsilon$, the contribution of this region to (13) is

$$
\frac{\delta N}{\bar{N}} \approx-\frac{8}{3 \pi(1-|s|)} \sqrt{\frac{m_{x}}{2 m_{y}}} \epsilon^{3 / 2} .
$$

There is no such contribution if $\nu$ is just above $1-|s|$, so there is a discontinuity in the density of states. While the precise prefactor and position of the discontinuity may depend on factors which we have not included, the threehalves power is generic, depending only on the topological property of having local minima of the integrand disappear. For example, in the event that $s<0$, we first lose the minimum around $\phi=0$ instead of $\phi=\pi / 2$ but otherwise the behaviour is the same and, except for a change in prefactor (typically it is smaller), we still expect a discontinuity of this power. Similarly, it is the same order of discontinuity at $\nu=1+|s|$ although, as stated, this is probably beyond the accessible magnetic field range.

To compare with experiment, we now determine the parameters of our model. The upper scale of $20 \mathrm{meV}$ equals $(1+s) \Delta_{0}$ and the lower scale of $5.5 \mathrm{meV}$ equals $(1-s) \Delta_{0}$ from which we infer $\Delta_{0} \approx 12.7 \mathrm{meV}$ and $s \approx$ 0.57 . We also use the result, consistent with both $\mu \mathrm{SR}$ 
and specific heat measurements that $E_{H} \approx 2.6 \sqrt{H}$ when $H$ is measured in Tesla and $E_{H}$ in meV [14].

The dimensionless parameter $\nu$ then equals $0.20 \sqrt{H}$. The dependence of the specific heat on $H$ has been measured in 15 18], with 18 having the cleanest samples. The data is shown in Fig. 3 in dimensionless units (taking the specific heat at low temperatures to be proportional to temperature and to the density of states.) By our previous arguments, energies of $5.5 \mathrm{meV}$ correspond to magnetic fields of about $4.5 T$ or $\nu$ of about 0.42 , which is well within the experimental ranges considered. The origin of the finite value of the density of states at zero field is not well understood but is sometimes attributed to oxygen vacancies on the chain [18] or to resonant impurity scattering [6]. Either way, to compare with our results one should subtract off this constant. There will also be a linearly increasing component from the density of states on the planes; this should be of comparable magnitude but without the lower singularity.
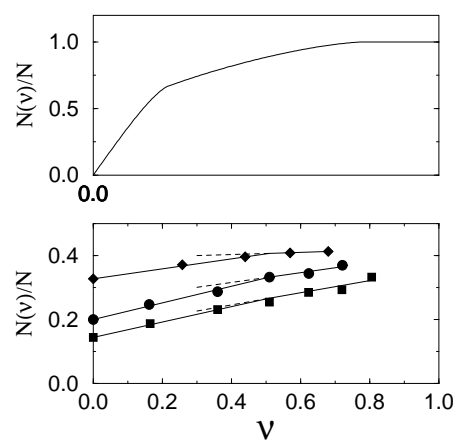

FIG. 3. Top: the density of states plotted against the rescaled magnetic field $\nu$ for the case $s=0.57$ and $m_{y} / m_{x}=0.5$. Bottom: experimental data converted to dimensionless units as described in the text. Circles, diamonds and squares are from $[15,16,18]$ respectively. In each case we have estimated a best-fit line below and above $\nu=0.5$ to guide the eye.

We plot this experimental data, fitting it with straight lines of different slopes at the two extremes of the data sets. There does appear to be a change in slope around $\nu=0.5$, in qualitative agreement with theory. While the theory does not predict a discontinuous slope, it does predict a nonanalyticity which resembles a change of slope. Since the data points are so sparse, we have not tried a detailed fitting to the predicted functional form. The magnitude of this nonanalyticity is given by the prefactor of the $\epsilon^{3 / 2}$ term in (16). (We note that the aforementioned background slope from the planes is present in the experimental data but not in the theoretical curve, so the relative magnitude of the discontinuity is different.) Experiments using a much finer sampling of magnetic field values would be required to verify this prediction.

In this paper we have considered a magnetic field parallel to the $c$-axis. In the case where it is parallel to the $a-b$ planes there may be additional interesting anisotropy effects [12]. Unfortunately, the energy scales seem to be such as to put this out of the experimentally accessible magnetic field range [20]. An interesting possibility is to consider a tilted magnetic field such that the energy scales are experimentally accessible while the inplane component is still strong enough to yield observable anisotropy effects. A gap function of mixed symmetry would have a clear signature on this anisotropy. Another interesting extension is to consider the role of the paramagnetic response of the electrons to the magnetic field 21] - an effect which has been completely neglected in the present work. While the energy scales seem to be such as to make this a reasonable assumption, it could well be that for the in-plane magnetic field, the paramagnetism is of comparable importance.

We thank I. Vekhter for useful discussions. Research supported in part by the Natural Sciences and Engineering Research Council (NSERC) and by the Canadian Institute for Advanced Research (CIAR).

[1] C. C. Tsuei and J. R. Kirtley, Physica C 282, 4 (1997).

[2] I. Maggio-Aprile et al., Phys. Rev. Lett. 75, 2754 (1995).

[3] Ch. Renner et al., Phys. Rev. Lett. 80, 3606 (1998).

[4] J. M. Valles et al., Phys. Rev. B 44, 11986 (1991).

[5] C. Caroli, P. G. de Gennes and J. Matricon, Phys. Lett. 9, 307 (1964).

[6] C. Kübert and P. J. Hirschfeld, Solid State Comm. 105, 459 (1998)

[7] A. G. Sun et al, Phys. Rev. Lett. 72, 2267 (1994); A. S. Katz et al., Appl. Phys. Lett. 66, 105 (1995); A. G. Sun et al., Phys. Rev. B 54, 6734 (1996); R. Kleiner et al., Phys. Rev. Lett. 76, 2161 (1996); J. Lesueur et al., Phys. Rev. B 55, R3398 (1997).

[8] K. A. Kouznetsov et al., Phys. Rev. Lett. 79, 3050 (1997).

[9] I. Schürer, E. Schachinger and J. P. Carbotte, Jour. Low Temp. Phys. 115, 251 (1999).

[10] C. O'Donovan and J. P. Carbotte, Phys. Rev. B 55, 1200 (1997); ibid 55, 8520 (1997).

[11] G. E. Volovik, JETP Lett. 58, 469 (1993).

[12] I. Vekhter et al., Phys. Rev. B 59, R9023 (1999).

[13] see for example, M. Tinkham, Introduction to Superconductivity, 2nd Ed., (McGraw-Hill, New York, 1996).

[14] I. Vekhter et al., Proceedings PPHMF-III, condmat/9811315.

[15] K. A. Moler et al., Phys. Rev. Lett. 73, 2744 (1994); Phys. Rev. B 55, 3954 (1997).

[16] R. A. Fisher et al., Physica C 252, 237 (1995).

[17] A. Junod et al., Physica C 282, 1399 (1997).

[18] D. L. Sisson et al., cond-mat/9904131.

[19] K. Maki and M. T. Beal-Monod, Phys. Rev. B 55, 11730 (1997).

[20] A. Junod, private communication.

[21] H. Won, H. Jang and K. Maki, cond-mat/9901252. 